\title{
3 Research Square \\ Potential Threats of Nanoplastic Accumulation in Human Induced Pluripotent Stem Cells
}

Hyejoong Jeong

Yonsei University

Wijin Kim

Kangwon National University

Daheui Choi

Yonsei University

Jiwoong Heo

Yonsei University

Uiyoung Han

Yonsei University

Se Yong Jung

Yonsei University College of Medicine

Hee Ho Park

Kangwon National University

Sung-Tae Hong

Seoul National University College of Medicine

Ju Hyun Park

Kangwon National University

Jinkee Hong ( $\sim$ jinkee.hong@yonsei.ac.kr)

Yonsei University https://orcid.org/0000-0003-3243-8536

\section{Research}

Keywords: Microplastic, Nanoplastic, Long-term toxicity, Bioaccumulation, Induced Pluripotent Stem Cell

Posted Date: April 27th, 2021

DOl: https://doi.org/10.21203/rs.3.rs-435972/v1

License: (9) This work is licensed under a Creative Commons Attribution 4.0 International License.

Read Full License 
Version of Record: A version of this preprint was published at Chemical Engineering Journal on January 1st, 2022. See the published version at https://doi.org/10.1016/j.cej.2021.131841. 


\section{Abstract}

Background: Micro- and nanoplastics (NPs) produced from the bulk fragmentation are rampant in the world by enormous plastic trash everyday life. NPs can be exposed to humans through a variety of routes, including inhalation and intake. The threat to humans from NPs is increasing invisibly. Nowadays, many people are concerned about human safety and health, but few are reported about the effects of NP on humans. To overcome the limitations in human studies, human induced pluripotent stem cells (hiPSCs) were used as an optimal in vitro platform to investigate developmental toxicology and subtle changes on cellular functions in terms of differentiation potential throughout a long-term culture.

Results: Negatively charged polystyrene nanoplastics (PS NPs) were used to exclude acute toxic issues of surface charge and investigate the impact of the NP's size and nature during bioaccumulation. Intracellular observations revealed that NPs up to $1000 \mathrm{~nm}$ were over-internalized into single cells within $48 \mathrm{~h}$, and smaller NPs demonstrated greater potency at decreasing number of viable cells by a strong correlation with the number of NPs on an equal mass basis. Also, PS NPs caused a significant reduction in self-renewal capacity of hiPSCs for $48 \mathrm{~h}$. After the cells were exposed to PS NPs for 48 to $96 \mathrm{~h}$ at the beginning of the differentiation process, NPs accumulated in hiPSC did not render cellular functions vulnerable or adversely affect EB formation, EB-mediated differentiation, and neural lineage differentiation for up to 14 days.

Conclusion: This study confirmed that hiPSC exposure to polystyrene nanoplastics results in acute toxicity and non-significant long-term effects on cellular functions. This report is important for understanding the developmental toxicology of nanoplastics and the origin of disease.

\section{Background}

Is it possible that microplastics (MPs) could cause life-threatening human diseases such as cancer? The answer is "no" based on our current understanding MPs, which are known to pervade the ecosystems, particularly oceans.[1] Plastic fragments $(<5 \mu \mathrm{m})$ are referred to as MPs and they are thought to be continuously degraded into 'nanoplastics (NPs)', i.e., fragments in the nanometer range (1 to $1000 \mathrm{~nm}$ ), via fragmentation and the weathering effect. $[2,3,4]$ NPs are too small to be eliminated using water purification systems and may be ingested by a wide range of organisms across the food chain.[5] of note, NPs are of particular concern as they can pass through biological membranes and cause abnormalities in cells.[6] Moreover, NPs with a large surface area are potentially hazardous as they readily adsorb contaminants, such as organic pollutants and heavy metals, and accumulate in living organisms. Finally, NPs are accumulated in humans via food, water from plastic containers, and inhalation. Indeterminate numbers of NPs would have already been exposed to humans.

The toxic effects of NPs on humans remain elusive due to the limited number of in vitro and in vivo studies.[7, 8] A number of short-term studies assessing NP toxicity using human cells have been reported. $[9,10,11,12]$ NPs generally exhibit very low toxicity due to their chemically stable nature and limited 
culture time. They tend to interact with the cell membrane and can translocate into the cell owing to their large surface area and physicochemical properties. $[13,14,15]$ Airborne particulate matter including silica nanoparticles, asbestos fibers, and coal particles can also access the cytosol because of their small size. [16] Such particles, when present in lung cells, induce inflammation and tissue damage eventually leading to the development of pulmonary fibrosis, mesothelioma, pneumoconiosis, and silicosis.[17, 18] Given that the nanoparticle accumulation in cells leads to the development of pathological conditions, cellular accumulation of NPs is also highly likely to be pathogenic. As reported by Gustafson et al., the initial disease phase follows patterns similar to those observed in acute toxicity studies in vitro.[18] Longterm in vitro studies are needed to evaluate the toxic effects of NPs on humans.

We selected human induced pluripotent stem cells (hiPSCs) as a toxicological platform to examine chronic impact of NPs in humans. Pluripotent stem cells (PSCs), such as embryonic stem cells (ESCs) and iPSCs, can be utilized as in vitro experimental models to study developmental toxicology due to their ability to differentiate into the three germ layers. The embryonic stem cell test (EST), which is an in vitro screening assay using mouse ESCs, - widely used as an animal free method- to assess embryotoxicity of reagents in vitro.[19, 20] PSCs are also suitable for assessing the long-term effects of exposure to toxic materials owing to their unlimited self-renewal capacity. Recent studies have explored the toxicological effects of metal nanoparticles on the viability, epigenetics, embryoid body (EB) formation, and differentiation potentials of ESCs.[21, 22] In addition, evidences for the translocation of plastic particles into fetal tissues have been shown. Fournier et al. has demonstrated in animal study that maternal pulmonary exposure to NPs lead to the accumulation of the NPs in both placental and fetal tissues.[23] Ragusa et al. has found the presence of multiple MPs in human placenta.[24] These results suggest the feasibility of using human PSCs as a model system to study the potential threat of NP accumulation in the early stages of human embryonic developments. In the present study, we focused on using negatively charged polystyrene nanoplastics (PS NPs) of various sizes (100 to $1000 \mathrm{~nm}$ ) to investigate the longterm impacts of size, excluding short-term toxicity caused by surface charge. Taking advantage of the colony-forming nature of hiPSC, we hypothesized that a larger number of smaller NPs would accumulate in the cells for a long time, resulting in functional changes in differentiation potentials. For comparison from a material point of view, $1000 \mathrm{~nm}$ of polyethylene microplastics (PE MPs) and mesoporous silica nanoparticles (MSNs) were used.

\section{Results And Discussion}

\section{Effect of NPs on hiPSC viability and self-renewal over the short term}

To assess the toxic effects of NPs on hiPSCs, we examined population of viable cells and undifferentiated state following NP exposure for up to $48 \mathrm{~h}$ (Fig. 1A). First, hiPSC colony formation was observed in response to PS NPs of different sizes, which were characterized based on size, morphology, dispersity, and surface charge (Fig. S1 and Table S1). PS NP precipitates were observed at the periphery of the colonies as small dark spots (Fig. 1B). Colonies maintaining their adherence and integrity exhibited absence of cell death. In addition, the result of alkaline phosphatase (AP) staining revealed that the 
undifferentiated state of hiPSCs were not significantly affected by NP exposure for up to $48 \mathrm{~h}$ (Fig. S2). Although there are no differences from the morphology of colonies and AP staining results, smaller colonies were observed the group treated smaller PS NPs (Fig. S3). The population of viable hiPSCsquantified using the WST-8 assay - was also reduced in a PS NP size and time-dependent manner (Fig. 1C), which is considered to be due to the growth inhibition by NP exposure, rather than cell death. This is because no significant alterations were observed in the morphology of the colonies. To date, the effect of NP size on toxicity is not clear, and different results have been reported depending on the cell type. Stock et al. analyzed the toxicity levels of 1, 4, and $10 \mu \mathrm{m}$ PS nanoparticles in Caco-2 cells and revealed pronounced cytotoxicity in response to small particles. When the effects of the following variables: number of particles, mass, and surface area were assessed, number of particles was found to exhibit the highest correlation with cytotoxicity.[14] Accordingly, number of NPs and surface area were assessed to discover the correlation with viable cell reduction rate (Fig. S4). The mass was not considered because the concentration of NPs was fixed. The viable cell reduction rate after $24 \mathrm{~h}$ presented a better correlation to the number of NPs. We verified number of NPs is the major factor determining the extent of cytotoxicity.

To evaluate the cytotoxic effect of the material, PS, PE, and MSN (size, $1000 \mathrm{~nm}$ ) were used. The most toxic material PS 100 (size, $100 \mathrm{~nm}$, units below are omitted.) was included as the positive control. Characterization of PE MPs and MSN is shown in Fig. S5 and Table S2. The colony formation and population of viable hiPSCs were investigated following treatments with each of the three NPs using a concentration range of $1-100 \mu \mathrm{g} / \mathrm{mL}$ for $48 \mathrm{~h}$. At the highest dose, none of the NPs negatively affected colony integrity as shown in Fig. 1D (no cell death). PE MPs and MSN did not exhibit formation of clearly observable agglomerates in the periphery of the colonies, compared with PS NPs, they were less likely to physically affect the colonies. Assessment of viable cell population revealed that only the MSN-treated group resulted in a slight reduction in population of viable cells (Fig. 1E). Collectively, the level of cytotoxicity was determined based on the type of material but the major factor affecting cytotoxicity was the size of NPs. The smaller NPs did not cause significant cell death in hiPSCs, but they may still exert toxic effects in the form of growth inhibition. As reported previously, cytotoxicity of MSN was presumably due to their efficient endocytosis. $[25,26]$

The maintenance of self-renewal capacity and undifferentiated state was verified after exposing hiPSCs to PS (PS 100 and PS $1000,100 \mu \mathrm{g} / \mathrm{mL}$ for $48 \mathrm{~h}$ ). Quantitative assessment of mRNA levels of Oct4 and Nanog-transcription factors indicative of pluripotent stem cells-revealed no change in Oct4 expression levels relative to those in control (Fig. 1F). However, a slight unexpected decrease in Nanog expression was detected. Oct4 is indispensable for maintaining the self-renewal and the pluripotency of hiPSCs.[27] For achieving an undifferentiated state, cells first overexpress Oct4, which subsequently induces the expression of all other factors involved in the pluripotency network, including Nanog.[28] Therefore, overexpression of Oct4 is less likely to reflect the effect of PS NPs. Nanog is also essential for attaining and maintaining self-renewal[29], it generally exhibits more fluctuations in expression than Oct4, and cells with low Nanog expression are prone to differentiation into three germ lineages.[27, 30] Owing to the 
heterogenous nature of Nanog, the observed decrease in Nanog mRNA levels in response to PS exposure is likely to reflect NP-induced reduction in self-renewal capacity.

\section{hiPSCs overloaded with NPs within 48 hours}

Generally, in normal cell types, internalized NPs are expelled via exocytosis, thereby limiting their cytosolic accumulation and consequent cellular damage.[31] However, it is thought that tight junctions between the hiPSCs in colonies may be responsible for the limited ability to perform exocytosis (Fig. 2A). Importantly, endocytosis can occur when hiPSCs that have not yet formed tight junctions are exposed to NPs. The colony state of hiPSC can be used as a toxicity testing platform for the bioaccumulation of NPs.

After exposure to NPs ( $100 \mu \mathrm{g} / \mathrm{mL}$ for $48 \mathrm{~h})$, the morphology and ultrastructure of the hiPSCs were observed using transmission electron microscopy (TEM) at a single cell level. NPs of all types, regardless of size and material, were clearly observed to form large clusters inside hiPSCs (Fig. 2B and Fig. S6 shows the control). PS 100 is likely to be incorrectly identified by readers as multivesicular bodies (MVBs). However, PS 100 can be distinguished from MVBs based on the presence of dozens of NPs in a vesicle and a regular shape with clear boundaries. Also, equal structures were found outside of the cells. Other PS NPs accumulated inside hiPSCs in a similar pattern, which makes them easily distinguishable from the cellular compartments and organelles.

According to a previous report, the uptake of nanoparticles is extremely limited in human embryonic stem cells (hESCs) compared to that in other cell types. This is usually attributed to the relatively compact and round structure of hESC colonies, which leaves little space for flexibility and limits the access of extracellular materials to the cytoplasm.[32] Senut et al. have explored the effect of gold nanoparticles (AuNPs) with three core sizes $(1.5,4$, and $14 \mathrm{~nm})$ on the viability and differentiation of hESCs, and found that only smallest AuNPs $(1.5 \mathrm{~nm})$ exhibited toxic effects on the viability and embryoid body (EB)mediated differentiation.[21] Intracellular uptake of both $4 \mathrm{~nm}$ and $14 \mathrm{~nm}$ AuNPs by hESCs was hardly detected, however at the same time occurred readily in mouse embryonic fibroblasts used as feeder cells. Interestingly, even $1.5 \mathrm{~nm}$ AuNPs, which were found to be highly toxic, was not taken up by hESCs, suggesting that $1.5 \mathrm{~nm}$ AuNPs imposed the cytotoxic effects without significant intracellular uptake. Another recent study has unveiled the effects of 40 and $200 \mathrm{~nm}$ PS NPs on the transcriptome of early human blastocysts hiPSCs.[33] Although it has been investigated which genes were upregulated or downregulated after PS NP exposure, clear evidence verifying the accumulation of NPs in hPSCs has not been provided. In this study, we found that all NPs were localized inside hiPSC regardless of their size, morphology, and nature. We believe that feeder-free culture protocol utilized in this study contributed to the accumulation of various PS NPs inside hiPSCs. Generally, hPSCs cultured with feeder cells form tightly packed colonies, which would not allow intracellular delivery of nanoparticles. On the other hand, feeder-free cultured hPSC colonies are less tight, especially in the periphery of the colony. The NPs were mainly localized in vesicles, including lysosomes, and the integrity of the cell membrane was maintained, suggesting that NPs were internalized by hiPSCs via cellular uptake. The pathway involved in the cellular internalization of nanoparticles is still not fully understood and depends on the cell type and nanoparticle 
characteristics (surface charge, size, and shape). In general, nanoparticles smaller than $200 \mathrm{~nm}$ are internalized via clathrin-mediated endocytosis, whereas caveolae-mediated internalization occurs as nanoparticle size increases.[34,35] Through this path, NPs did not significantly damage the organelles or affect cellular integrity. In addition, the size of vesicles containing nanoparticles increased through fusion. and the vesicle with PS 1000 has been shown to occupy over $70 \%$ of the total cell area. Another notable point is that the accumulation of PS in the lysosomes can induce osmotic flow and lysosomal swelling. Recent studies have reported that the presence of large clusters of nondegradable gold nanoparticles-which are eliminated by cancer cells-caused gradual loss in lysosomal membrane integrity, and finally, cell death in 48 h.[36] The present results also indicated lysosomal swelling owing to NP overload, potential toxicity remains. Previous reports have demonstrated that inert and biopersistent nanoparticles do not cause acute toxicity, but may be of greater concern, as they can block essential cellular functions, including cytoskeleton formation, cellular movement, and exocytosis. [13, 14, 35, 37]

The results of TEM prompted us to further examine the uptake of PS by cells in the colonies. Confocal microscopy was attempted to confirm the distribution of NPs in the colonies. First, hiPSC colonies exposed to PS 100, 500, and 1000 for 24 h were observed after fixation (Fig. 2C, Fig. S7). PS 100 was distributed throughout the colony (indicated by white arrows), whereas PS 500 and PS 1000 were observed only at the rim of the colonies and scarcely penetrated the center. The central cells are relatively compact due to the presence of tight junctions and are pressured by the marginal cells that create a tight iPSC colony structure. Furthermore, this pressure increases during colony growth and internalization of exogenous materials is scarce.[38] Consequently, large PS NPs were mostly found in cells at the rim. Significantly, when taken into account with the viable cell population results for $48 \mathrm{~h}$, higher internalization efficiency throughout the colonies may affect viable cell population reduction (Fig. 1C).

Further Z-stack imaging revealed that all PS were localized in the intracellular region but were rarely located in the extracellular area (Fig. S8-S10). In addition, to eliminate the possible effect of fixation on NP uptake, the colonies were also observed in the living state, and identical results were obtained (Fig. S11).

\section{Long-term effect of NPs-laden hiPSCs on differentiation potency}

The long-term impact of NP accumulation in hiPSCs was investigated by monitoring both EB-mediated and neural differentiation. Colonies exposed to NPs (PS 100, PS 1000, PE 1000, and MSN 1000) for $48 \mathrm{~h}$ were dissociated into single cells, and subsequently allowed to form EBs for 14 days in suspension culture, whereas, the other hiPSCs exposed to PS (100 and $1000 \mathrm{~nm})$ were directly differentiated into neural stem cells (NSCs) for 7 days in adherent culture (Fig. 3A).

After 14 days, EBs of all groups maintained their morphology without any abnormalities compared to those in the control group (Fig. 3B). To evaluate the differentiation potency of EBs, the expression of the markers of the three germ layers was analyzed using quantitative real-time polymerase chain reaction (qPCR) (Fig. 3C). Oct4, PAX6, Sox17, and TAL 1 mRNA represent pluripotency, ectoderm, endoderm, and mesoderm markers, respectively. First, successful EB-mediated differentiation was confirmed based on 
the low expression of Oct4. The mRNA expression of the three germ layer markers was simultaneously upregulated in a similar manner in all groups, except Sox 17 for MSN 1000. Taken together, the mRNA expression of lineage-specific markers suggests that none of the NPs significantly affected spontaneous differentiation via EB formation.

We then evaluated the neural differentiation capacity of hiPSCs exposed to NPs without forming EBs. PS 100 and PS 1000 were used to examine the toxic effects of NPs on neural differentiation. After the pretreatment of hiPSCs with each group of PS for 48 and $96 \mathrm{~h}$ and subsequent neural differentiation for 7 days, the typical neural tube-like rosettes of NSCs were successfully formed[39] and maintained in a state identical to those in the non-treated group (Fig. 3D). The dark colonies in the PS 1000-treated groups compelled us to propose that the dark color could be attributed to the agglomerations of the large-sized NPs inside the cells. Further, quantitative analysis of ectoderm markers reconfirmed the formation of NSCs (Fig. 3E). Compared to the hiPSCs, all groups exhibited low expression of Oct4, indicating successful differentiation. The PS NPs-treated groups did not exhibit a difference in the expression of ectoderm markers PAX6 or ZIC1.

Collectively, several important conclusions can be reached as summarized in Fig. 4:

(a) Internalization and accumulation of NPS: A large amount of all types of NPs was internalized into hiPSCs within $48 \mathrm{~h}$. Smaller PS were efficiently located not only in the cells at the rim, but also in cells at the center of colonies. The PS NPs were accumulated in the hiPSCs due to the cell-cell tight junctions of colonies.

(b) Growth inhibition: The smaller PS NPs resulted in severe growth inhibition in hiPSCs as large numbers were internalized into cells. Colonies exposed to NPs maintained their integrity, but colony size was reduced due to the growth inhibitory effect of smaller PS.

(c) Reduction of self-renewal capacity. PS NPs (PS 100 and PS 1000) did not significantly affect colony formation but are likely to reduce the self-renewal capacity of hiPSCs.

(d) No long-term impacts on differentiation: None of the NPs tested (PS 100, PS 1000, PE 1000, and MSN 1000) affected the formation or differentiation potential of EBs for 14 days. PS NPs (PS 100 and PS 1000) did not significantly affect the differentiation of hiPSCs into NSCs for 7 days.

\section{Conclusion}

We demonstrated size-dependent internalization of PS NPs in hiPSC and the impacts on self-renewal capacity and pluripotency. Evaluation of viable cell concentration and mRNA expression of undifferentiated state markers revealed that short-term exposure to smaller PS NPs for up to $48 \mathrm{~h}$ lead to reduction in self-renewal capacity of hiPSCs. Concurrently, ultrastructure and confocal images identified a larger number of PS NPs internalization in single cells and colonies. The internalized PS NPs in the cytosol and throughout the colonies were demonstrated to be associated with their toxic effects. 
Although no significant impacts on the differentiation potential of hiPSCs were shown in the in vitro differentiation for up to 2 weeks, the large-scale intracellular accumulation of NPs with mean diameter of up to $1000 \mathrm{~nm}$ in hiPSCs suggests the possibility of causing unexpected alterations in human embryogenesis in which sophisticated differentiation processes occur simultaneously. Previous studies showing evidence of the translocation of NPs from maternal to fetal tissues across the placenta support this hypothesis. Despite the recent advances in the understanding of plastic toxicology, the impacts of NP exposure on human health, especially embryogenesis, have still remained elusive. To the best of our knowledge, this is the first study to demonstrate long-term toxicity assessment of NPs using hiPSCs. We believe that an important finding of this study is to draw attention to the potential threat of maternal NP exposure, which might interfere with human embryogenesis, by demonstrating the accumulation of NPs inside hiPSCs. Furthermore, we expect that the hiPSC will be a versatile platform to reveal chronic impacts of a variety of NPs which are contaminated, positively charged, and other types of materials.

\section{Materials And Methods}

\section{Materials}

Polystyrene nanoplastics (PS NPs, sizes: 100, 200, 500, $1000 \mathrm{~nm}$ ) and mesoporous silica microparticle (MSNs) $1000 \mathrm{~nm}$ in diameter with $2 \mathrm{~nm}$ of pore size were purchased from Sigma-Aldrich (MO, USA). Polyethylene nanoplastics (PE MPs, sizes 1 to $10 \mu \mathrm{m}$ ) were purchased from Cospheric (CA, USA). For the confocal observation, negatively charged fluorescent polystyrene nanoplastics (FL-PS NPs, sizes: 100, $500,1000 \mathrm{~nm}$ ) were obtained from Sigma-Aldrich (MO, USA).

Prior to cell treatment, the particles were sterilized with ethylene oxide gas.

\section{Particles Characterization}

The morphologies of the NPs were observed using a scanning electron microscopy (SEM, JEOL-7610FPlus, Japan) and a transmission electron microscopy (TEM, JEM1011, JEOL, Japan). The size of the particles was analyzed based on the SEM images and ImageJ software. The size distribution was defined as the coefficient of variation (Dispersity $(\%)=$, where $\sigma$ is the standard deviation and $d$ is the mean size. The surface charge of particles was measured by a $\xi$-potential in distilled water (SZ-100, Horiba, Japan). Hydrodynamic size $\left(D_{H}\right)$ of particles was measured by dynamic light scattering using equal equipment with $\xi$-potential. The concentration of particles for $\xi$-potential and $D_{H}$ measurement was $0.1 \mathrm{mg} / \mathrm{mL}$.

\section{Cell Culture}

Human induced pluripotent stem cells (hiPSCs) used in this study were obtained from National Center for Stem cell and Regenerative Medicine in Korea. This iPSC line was generated by introducing four reprogramming factors (Oct4, c-Myc, Klf4, and Sox2) into human dermal fibroblasts using Sendai virus. hiPSCs were cultured in TeSR ${ }^{\mathrm{TM}}$-E8 ${ }^{\mathrm{TM}}$ medium (E8 medium, STEMCELL Technologies, Vancouver, 
Canada). When cell confluency reached $80 \%$, cells were detached by $0.5 \mathrm{mM}$ EDTA treatment and plated onto a Matrigel ${ }^{\mathrm{TM}}$ (Corning, NY, USA)-coated dish.

\section{Determination of viable cell population by WST-8 Assay}

Water-soluble tetrazolium salt-8 (WST-8) assay was performed using Quanti-MAX ${ }^{\mathrm{TM}}$ WST-8 Cell Viability Assay Kit (Biomax Co. Ltd., Korea) according to the manufacturer's instruction. hiPSCs were plated as single cells at $0.510^{4} \mathrm{cell} /$ / in 96 -well plate and incubated at $37^{\circ} \mathrm{C}$ with $5 \% \mathrm{CO} \otimes$ condition. After 3 days, all NPs were treated for up to 2 days. To determine viability, the cells were washed with Dulbecco's phosphate-buffered saline (DPBS) and incubated for $2 \mathrm{~h}$ with the addition of $10 \mu \mathrm{l} \mathrm{WST-8}$ solution. Absorbance was measured at $450 \mathrm{~nm}$ using a spectrophotometer. Based on the measured absorbance values, the survival rate [\%] of the cells is calculated by the following equation.

$$
\text { Population of viable cells }[\%]=\left(1-\frac{\text { Absorbance of NPs treated group }}{\text { Absorbance of the control }}\right) \times 100
$$

\section{Embryoid Body (EB) Formation}

To evaluate the developmental toxicity, hiPSCs exposed to each MP were allowed to form embryoid body (EB) and spontaneously differentiated into various lineages. Prior to form EB, hiPSCs were exposed to NPs for 2 days. Afterward, hiPSCs were dissociated to single cells by mild pipetting after EDTA treatment and plated on Ultra-low attachment 24-well plate (flat-bottom, Corning) at $210^{4}$ cells/ $\mathbb{\text { . }}$. Cells were suspended and further cultured for 14 days in AggreWell ${ }^{\mathrm{TM}}$ EB Formation Medium (STEMCELL Technologies). During the first 2 days of culture, $10 \mu \mathrm{M}$ Y-27632, a specific inhibitor for Rho-dependent proteins kinase (ROCK), was supplemented to the medium to block the single cell dissociation-induced apoptosis. Then, the medium was replaced every 2 days with a fresh medium.

\section{PCR Analysis}

The mRNA expression levels were determined by quantitative real time polymerase chain reaction (qPCR). Total RNA was extracted using Ribospin ${ }^{\mathrm{TM}}$ total RNA purification kit (GeneAll Biotechnology CO, Ltd, Korea) according to manufacturer's instructions. Then, cDNA was synthesized from the purified total RNA by reverse transcription using TOPScript ${ }^{\mathrm{TM}} \mathrm{RT}$ DryMIX (Enzynomics, Korea) with a dT 18 plus primer. qPCR was performed using the synthesized cDNA, TOPreal ${ }^{\mathrm{TM}}$ QPCR 2PreMIX (SYBR Green with low ROX, Enzynomics) and specific primers on the Eco Real-Time PCR System (Illumina, CA, USA). The expression level of each mRNA was normalized by glyceraldehyde 3-phosphate dehydrogenase (GAPDH) as an endogenous control, and the relative expression level, compared to undifferentiated iPSCs, was evaluated by the $2^{-\Delta \Delta C t}$ method.

\section{Electron Microscopy and Ultrastructural Analysis}


hiPSCs exposed to all NPs for $48 \mathrm{~h}$ were fixed over $12 \mathrm{~h}$ in $2 \%$ glutaraldehyde-paraformaldehyde in $0.1 \mathrm{M}$ phosphate buffer ( $\mathrm{pH} 7.4$ ) and washed with $0.1 \mathrm{M}$ phosphate buffer (PB). Afterward, the cells were postfixed with $1 \%$ osmium tetroxide $\left(\mathrm{OsO}_{4}\right)$ dissolved in $0.1 \mathrm{M} \mathrm{PB}$ for $2 \mathrm{~h}$ and dehydrated with series of the graded ethyl alcohol (50 100\%) and infiltrated with propylene oxide. The specimens were embedded by Poly/Bed 812 kit (Polysciences, PA) and the polymerization was performed at $65^{\circ} \mathrm{C}$ electron microscope oven (TD-700, DOSAKA, Japan) for $24 \mathrm{~h}$. After cutting the specimens to 200 250 nm thick and stained with toluidine blue (Sigma, St. Louis, MO) for optical microscope. Then the specimens were sliced to ultrathin sections with $70 \mathrm{~nm}$ thick by ultramicrotome (LEICA EM UC-7, Leica Microsystems, Austria) and double stained with $6 \%$ uranyl acetate for 20 min (EMS, Hatfield, PA) and lead citrate for 10 min (ThermoFisher, Waltham, MA) for contrast staining. The ultrathin sections, transferred on copper and nickel grids, were observed by transmission electron microscopy (JEM1011, JEOL, Japan) at the

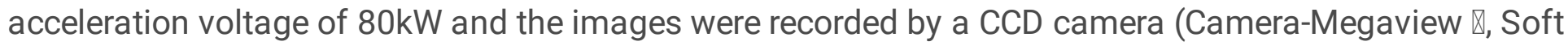
Imaging System GmbH, Germany).

\section{Confocal Microscopy Images}

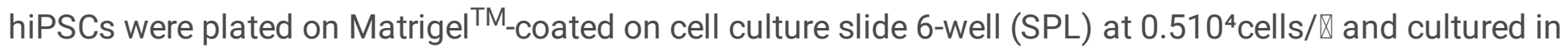
3 days at $37^{\circ} \mathrm{C}$ and $5 \% \mathrm{COQ}$. Cells were treated with fluorescent labeled PS NPs for $24 \mathrm{~h}$ in E8 medium. After incubation, the cells were washed with DPBS 3 times and fixed with $4 \%$ of paraformaldehyde for 15 min. Afterward the cells were incubated with 4',6-diamidino-2-phenylindole (DAPI, Sigma-Aldrich, MO, USA) for $1 \mathrm{~min}$. Images were obtained from confocal laser scanning microscope (CLSM) (LSM880 with Airyscan, Carl Zeiss, Germany). For the live cell observation, the samples were prepared following the identical method with above excluding the cell fixation process and images were obtained by inverted microscope (DMi8, Leica, Germany)

\section{Neural Differentiation}

hiPSCs were plated on 6-well plate coated by Matrigel ${ }^{\mathrm{TM}}$ at $0.510^{4} \mathrm{cells} / \mathrm{cm}^{2}$ and cultured at $37^{\circ} \mathrm{C}$ and $5 \%$ CO\&. hiPSCs were exposed to $100 \mu \mathrm{g} / \mathrm{mL}$ of both 100 and $1000 \mathrm{~nm}$ PS NPs dispersed in E8 medium from day 1 for $96 \mathrm{~h}$ and from day 3 for $48 \mathrm{~h}$, respectively, with daily medium replacement. At day 5 of culture, hiPSCs were dissociated as single cells and plated on 12-well plate coated by Matrigel at $110^{4} \mathrm{cell} / \mathrm{sm}$ in the presence of $10 \mu \mathrm{M} \mathrm{Y}-27632$. After incubation at $37^{\circ} \mathrm{C}$ and $5 \% \mathrm{CO} \otimes$ for $24 \mathrm{~h}$, the medium was replaced with PSC Neural Induction Medium (Thermo Scientific, MA, USA). During the neural differentiation, the cells were cultured for 7 days by replacing the medium every 2 days with fresh medium.

\section{Statistical Analysis}

Statistical analysis was performed to verify statistical significance between a negative control and treated groups using one-way ANOVA. Data values were provided as meanstandard deviation (SD). All quantitative results were obtained from triplicate samples in a representative of experiments performed several times. 


\section{Abbreviations}

NP, nanoplastic, hiPSC, human induced pluripotent stem cell, PS, polystyrene, PE, polyethylene, MSN, mesoporous silica nanoplastic, EB, embryonic body, PCR, polymerase chain reaction, NSC, neural stem cell, TEM, transmission electron microscopy, hESC, human embryonic stem cell, MVB, multivesicular bodies.

\section{Declarations}

\section{Acknowledgements}

Not applicable.

\section{Author's contributions}

$\ddagger$ These authors contributed equally. H.J., W.K., D.C., J.H., U.H., H.H.P., J.H.P., and J.H. analyzed the results. H.J. and W.K. performed the experiments. H.J., S.Y.J., J.H.P., and J.H. performed further analysis. H.J., W.K., S.Y.J., S-T. H., J.H.P., and J.H. wrote and edited the manuscript.

\section{Funding}

This research was supported by the Bio \& Medical Technology Development Program of the National Research Foundation (NRF) funded by the Ministry of Science and ICT of the Korean government (2016M3A9C6917402, 2016M3A9C6917405, 2019M3A9H110378611). In addition, this research was also supported by the Basic Science Research Program through the NRF (NRF-2017R1E1A1A01074343, 2020R1A4A1016093).

\section{Availability of data and materials}

The datasets used and/or analyzed during the current study are available from the corresponding author on reasonable request.

\section{Ethics approval and consent to participate}

Not applicable.

\section{Consent for publication}

All authors have read and approved the manuscript. The need for publication is not applicable.

\section{Competing interests}

Authors declare no competing interests.

\section{Author details}


${ }^{1}$ Department of Chemical and Biomolecular Engineering, Yonsei University, Seoul 03722, Republic of Korea, ${ }^{2}$ Department of Biomedical Science, Kangwon National University, Chuncheon, Gangwon-do, 24341, Republic of Korea, ${ }^{3}$ Department of Pediatrics, Yonsei University College of Medicine, Seoul 03722, Republic of Korea, ${ }^{4}$ Department of Biotechnology and Bioengineering, Kangwon National University, Chuncheon, Gangwon-do 24341, Republic of Korea, ${ }^{5}$ Department of Tropical Medicine and Parasitology, Seoul National University College of Medicine, Seoul 03080, Republic of Korea.

\section{References}

1. Ivar do Sul JA, Costa MF. The present and future of microplastic pollution in the marine environment. Environ Pollut. 2014;185:352-64.

2. Koelmans AA, Diepens NJ, Velzeboer I, Besseling E, Quik JTK, van de Meent D. Guidance for the prognostic risk assessment of nanomaterials in aquatic ecosystems. Sci Total Environ. 2015;535:141-9.

3. Kooi M, Nes EHv, Scheffer M, Koelmans AA. Ups and downs in the ocean: effects of biofouling on vertical transport of microplastics. Environ Sci Technol. 2017;51:7963-71.

4. da Costa JP, Santos PS, Duarte AC, Rocha-Santos T. (Nano) plastics in the environment-sources, fates and effects. Sci Total Environ. 2016;566:15-26.

5. Moore CJ. Synthetic polymers in the marine environment: A rapidly increasing, long-term threat. Environ Res. 2008;108:131-9.

6. Bergmann M, Gutow L, Klages M. Marine anthropogenic litter. Springer, 2015.

7. Revel M, Châtel A, Mouneyrac C. Micro (nano) plastics: A threat to human health? A. Curr Opin Environ Sci Health. 2018;1:17-23.

8. Waring R, Harris R, Mitchell S. Plastic contamination of the food chain: A threat to human health? Maturitas. 2018;115:64-8.

9. Hwang J, Choi D, Han S, Jung SY, Choi J, Hong J. Potential toxicity of polystyrene microplastic particles. Sci Rep. 2020;10:7391.

10. Xu M, Halimu G, Zhang Q, Song Y, Fu X, Li Y, et al. Internalization and toxicity: A preliminary study of effects of nanoplastic particles on human lung epithelial cell. Sci Total Environ. 2019;694:133794.

11. He Y, Li J, Chen J, Miao X, Li G, He Q, et al. Cytotoxic effects of polystyrene nanoplastics with different surface functionalization on human HepG2 cells. Sci Total Environ. 2020;723:138180.

12. Cortés C, Domenech J, Salazar M, Pastor S, Marcos R, Hernández A. Nanoplastics as a potential environmental health factor: Effects of polystyrene nanoparticles on human intestinal epithelial Caco-2 cells. Environ Sci: Nano. 2020;7:272-85.

13. Wu B, Wu X, Liu S, Wang Z, Chen L. Size-dependent effects of polystyrene microplastics on cytotoxicity and efflux pump inhibition in human Caco-2 cells. Chemosphere. 2019;221:333-41.

14. Stock V, Böhmert L, Lisicki E, Block R, Cara-Carmona J, Pack LK, et al. Uptake and effects of orally ingested polystyrene microplastic particles in vitro and in vivo. Arch Toxicol. 2019;93:1817-33. 
15. Heinlaan M, Kasemets K, Aruoja V, Blinova I, Bondarenko O, Lukjanova A, et al. Hazard evaluation of polystyrene nanoplastic with nine bioassays did not show particle-specific acute toxicity. Sci Total Environ. 2020;707:136073.

16. Byrne JD, Baugh JA. The significance of nanoparticles in particle-induced pulmonary fibrosis. McGill J Med. 2008;11:43.

17. Fujimura N. Pathology and pathophysiology of pneumoconiosis. Curr Opin Pulm Med. 2000;6:1404.

18. Gustafson HH, Holt-Casper D, Grainger DW, Ghandehari H. Nanoparticle uptake: the phagocyte problem. Nano Today. 2015;10:487-510.

19. Seiler AE, Spielmann $H$. The validated embryonic stem cell test to predict embryotoxicity in vitro. Nat Protoc. 2011;6:961-78.

20. Jung EM, Choi Yu, Kang HS, Yang H, Hong EJ, An BS, et al. Evaluation of developmental toxicity using undifferentiated human embryonic stem cells. J Appl Toxicol. 2015;35:205-18.

21. Senut MC, Zhang Y, Liu F, Sen A, Ruden DM, Mao G. Sizedependent toxicity of gold nanoparticles on human embryonic stem cells and their neural derivatives. Small 2016 12:631-646.

22. Zhang J, Chen Y, Gao M, Wang Z, Liu R, Xia T, et al. Silver nanoparticles compromise female embryonic stem cell differentiation through disturbing $X$ chromosome inactivation. ACS Nano. 2019;13:2050-61.

23. Fournier SB, D'Errico JN, Adler DS, Kollontzi S, Goedken MJ, Fabris L, et al. Nanopolystyrene translocation and fetal deposition after acute lung exposure during late-stage pregnancy. Part Fibre Toxicol. 2020;17:55-66.

24. Ragusa A, Svelato A, Santacroce C, Catalano P, Notarstefano V, Carnevali O, et al. Plasticenta: First evidence of microplastics in human placenta. Environ Int. 2021;146:106274.

25. Tao Z, Toms BB, Goodisman J, Asefa T. Mesoporosity and functional group dependent endocytosis and cytotoxicity of silica nanomaterials. Chem Res Toxicol. 2009;22:1869-80.

26. Petushkov A, Ndiege N, Salem AK, Larsen SC. Toxicity of silica nanomaterials: zeolites, mesoporous silica, and amorphous silica nanoparticles. Adv Mol Toxicol. 2010;4:223-66.

27. Olariu V, Lövkvist C, Sneppen K. Nanog, Oct4 and Tet1 interplay in establishing pluripotency. Sci Rep. 2016;6:25438.

28. Loh Y-H, Wu Q, Chew J-L, Vega VB, Zhang W, Chen X, et al. The Oct4 and Nanog transcription network regulates pluripotency in mouse embryonic stem cells. Nat Genet. 2006;38:431-40.

29. Chambers I, Silva J, Colby D, Nichols J, Nijmeijer B, Robertson M, et al. Nanog safeguards pluripotency and mediates germline development. Nature. 2007;450:1230-4.

30. Kalmar T, Lim C, Hayward P, Munoz-Descalzo S, Nichols J, Garcia-Ojalvo J, et al. Regulated fluctuations in nanog expression mediate cell fate decisions in embryonic stem cells. PLoS Biol. 2009;7:e1000149. 
31. Oh N, Park J-H. Endocytosis and exocytosis of nanoparticles in mammalian cells. Int J Nanomed. 2014;9:51-63.

32. Thomson JA, Itskovitz-Eldor J, Shapiro SS, Waknitz MA, Swiergiel JJ, Marshall VS, et al. Embryonic stem cell lines derived from human blastocysts. Science. 1998;282:1145-7.

33. Bojic S, Falco MM, Stojkovic P, Ljujic B, Gazdic Jankovic M, Armstrong L, et al. Platform to study intracellular polystyrene nanoplastic pollution and clinical outcomes. Stem Cells. 2020;38:1321-5.

34. Rejman J, Oberle V, Zuhorn IS, Hoekstra D. Size-dependent internalization of particles via the pathways of clathrin-and caveolae-mediated endocytosis. Biochem J. 2004;377:159-69.

35. Seydoux E, Rothen-Rutishauser B, Nita IM, Balog S, Gazdhar A, Stumbles PA, et al. Size-dependent accumulation of particles in lysosomes modulates dendritic cell function through impaired antigen degradation. Int J Nanomed. 2014;9:3885-902.

36. Borkowska M, Siek M, Kolygina DV, Sobolev YI, Lach S, Kumar S, et al. Targeted crystallization of mixed-charge nanoparticles in lysosomes induces selective death of cancer cells. Nat Nanotech. 2020;15:331-41.

37. Huang $X$, Teng $X$, Chen $D$, Tang F, He J. The effect of the shape of mesoporous silica nanoparticles on cellular uptake and cell function. Biomaterials. 2010;31:438-48.

38. Hammerick KE, Huang Z, Sun N, Lam MT, Prinz FB, Wu JC, et al. Elastic properties of induced pluripotent stem cells. Tissue Eng Part A. 2011;17:495-502.

39. Hu B-Y, Weick JP, Yu J, Ma L-X, Zhang X-Q, Thomson JA, et al. Neural differentiation of human induced pluripotent stem cells follows developmental principles but with variable potency. Proc Natl Acad Sci. 2010;107:4335-40.

\section{Figures}



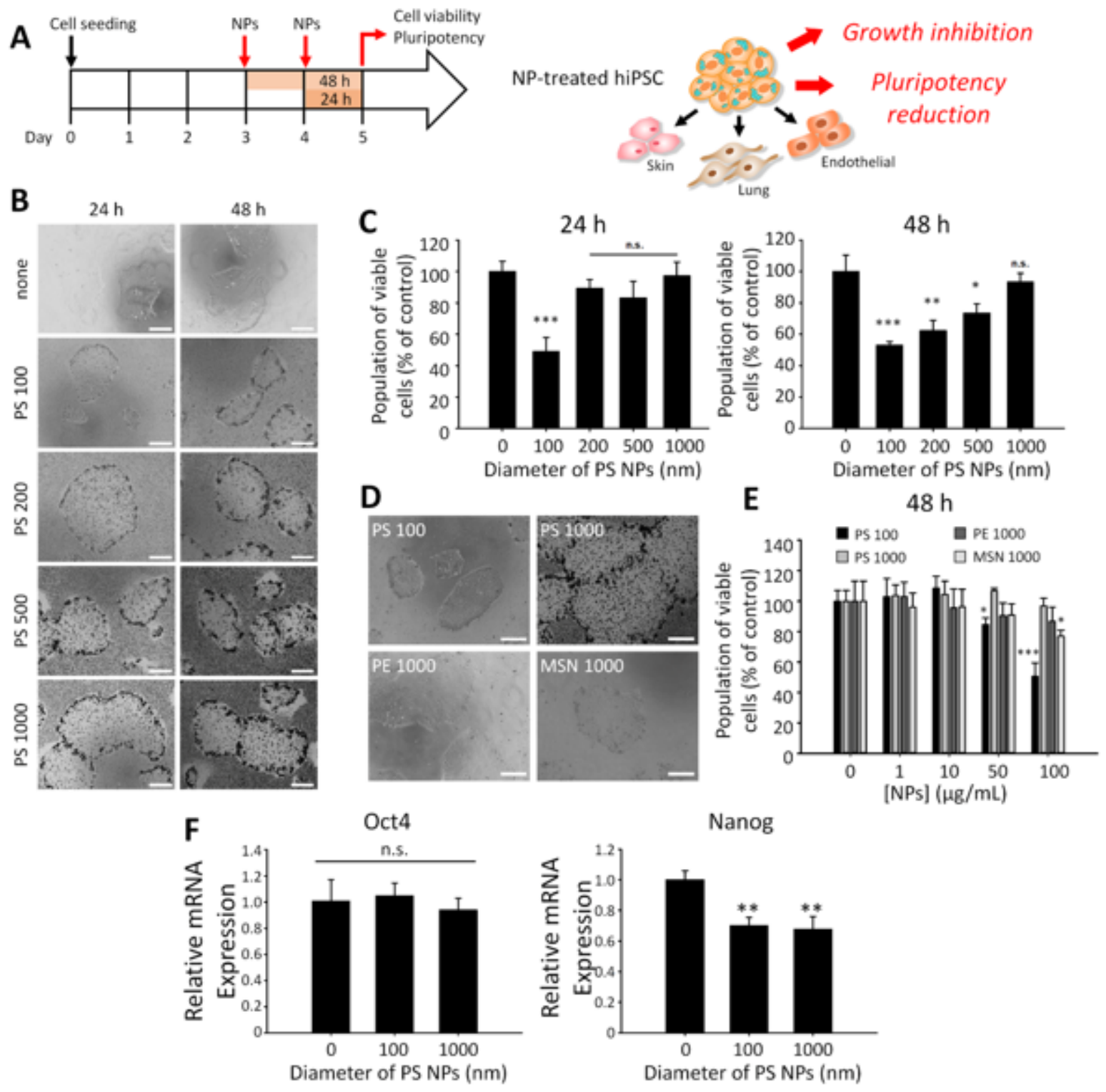

\section{Figure 1}

Cytotoxic effects of NPs (PS, PE MPs, and MSN) on hiPSCs. PS NPs, polystyrene nanoplastics, PE MPs, polyethylene microplastics, MSN, mesoporous silica nanoparticles, hiPSCs, human induced pluripotent stem cells. (A) Schematic illustration of the experiment and the effects of NPs on hiPSCs. (B) Optical images of feeder-free-cultured hiPSC colonies following exposure to the different sizes of PS NPS (ranging from 100 to $1000 \mathrm{~nm}$, up to $48 \mathrm{~h}, 100 \mu \mathrm{g} / \mathrm{mL}$ ). The scale bar represents $200 \mu \mathrm{m}$. (C) Viability of (B) measured by WST-8 assay. The percentage of viable cells was compared to that in the non-treated control $(0 \mu \mathrm{g} / \mathrm{mL})$. $(n=3)(D)$ Observation of feeder-free hiPSC colonies following exposure to PS 100 , PS 1000, PE 1000, and MSN $1000 \mathrm{~nm}(100 \mu \mathrm{g} / \mathrm{mL}, 48 \mathrm{~h})$. The scale bar represents $200 \mu \mathrm{m}$. (E) Viability of (D) measured by WST-8 assay. The percentage of viable cells was compared to that in the non-treated control $(0 \mu \mathrm{g} / \mathrm{mL})$. $(\mathrm{n}=3)(\mathrm{F})$ Verification of the self-renewal marker (Oct4 and Nanog) expression based on mRNA expression (100 and $1000 \mathrm{~nm}, 100 \mu \mathrm{g} / \mathrm{mL}, 48 \mathrm{~h}$ ). The control group represents 0 (non-treated). 
Histogram values are mean $\pm S D$. $(n=3)$ Significance compared to the non-treated group is noted as *, $p$ $\leq 0.05, * *, p \leq 0.15$, and $* * *, p \leq 0.001$ ( $n=3$, one-way ANOVA). n.s. indicates non-significant.
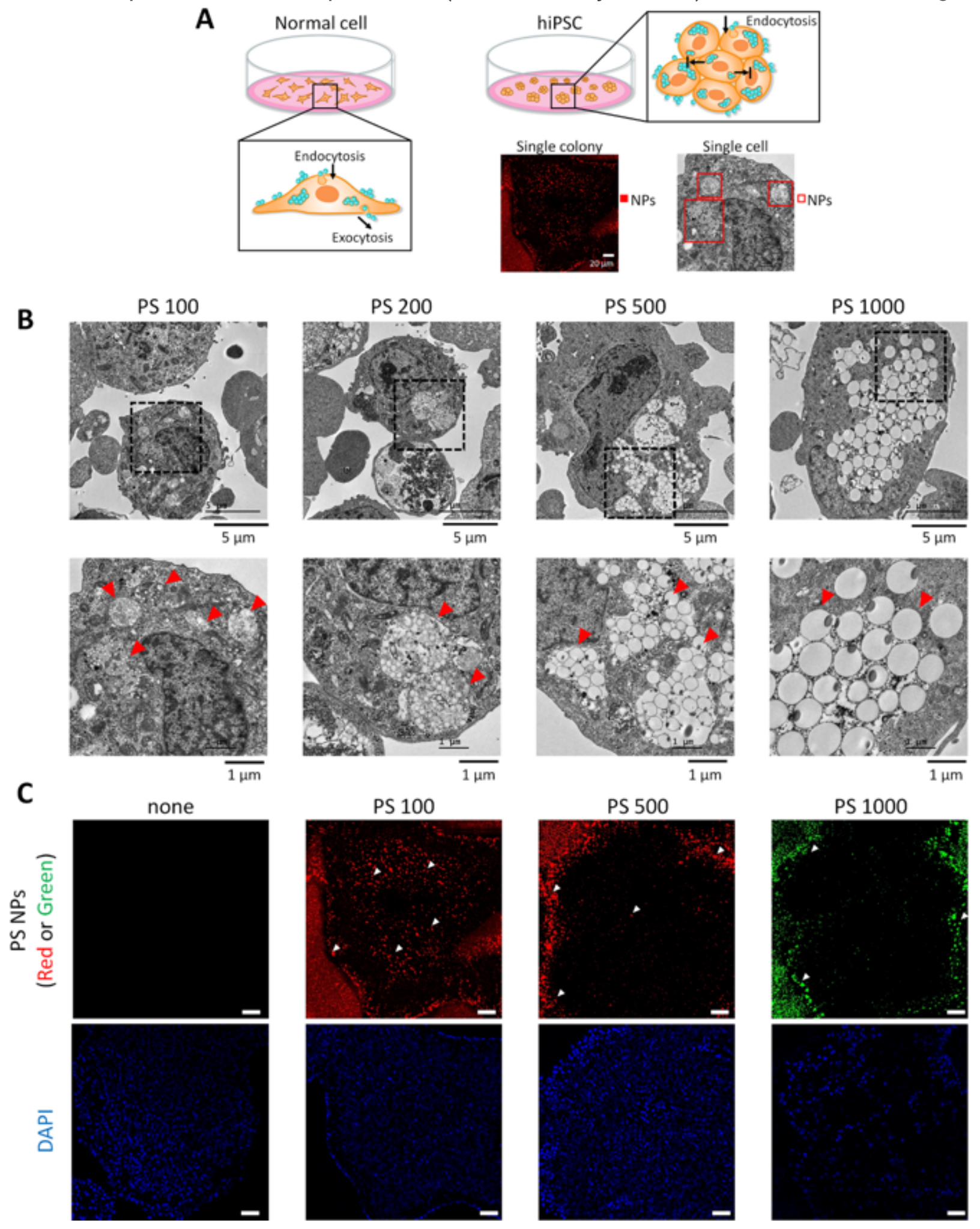

Figure 2

PS NPs localized inside hiPSC colonies and cells. PS NPs, polystyrene nanoplastics, hiPSCs, human induced pluripotent stem cell, TEM, transmission electron microscopy. (A) Schematic illustration of the functional difference between normal cells and hiPSCs. (B) Ultrastructure of hiPSCs observed using TEM 
after exposure to PS NPs (PS 100, 200, 500, 1000, units are $\mathrm{nm}, 100 \mu \mathrm{g} / \mathrm{mL}, 48 \mathrm{~h}$ ). A specific part of the TEM image (dotted square) is enlarged and shown below. The red arrows indicate the cluster of PS NPs. (C) Distribution of PS NPs in hiPSC colonies exposed to $100 \mu \mathrm{g} / \mathrm{mL}$ of PS NPs (100, 500 and $1000 \mathrm{~nm}$ ) for $24 \mathrm{~h}$. PS 100 and 500 exhibited red fluorescence and PS 1000 showed green fluorescence. Nuclei were stained with DAPI (blue). White arrows indicate PS NPs located in the intracellular region. Scale bar represents $75 \mu \mathrm{m}$.
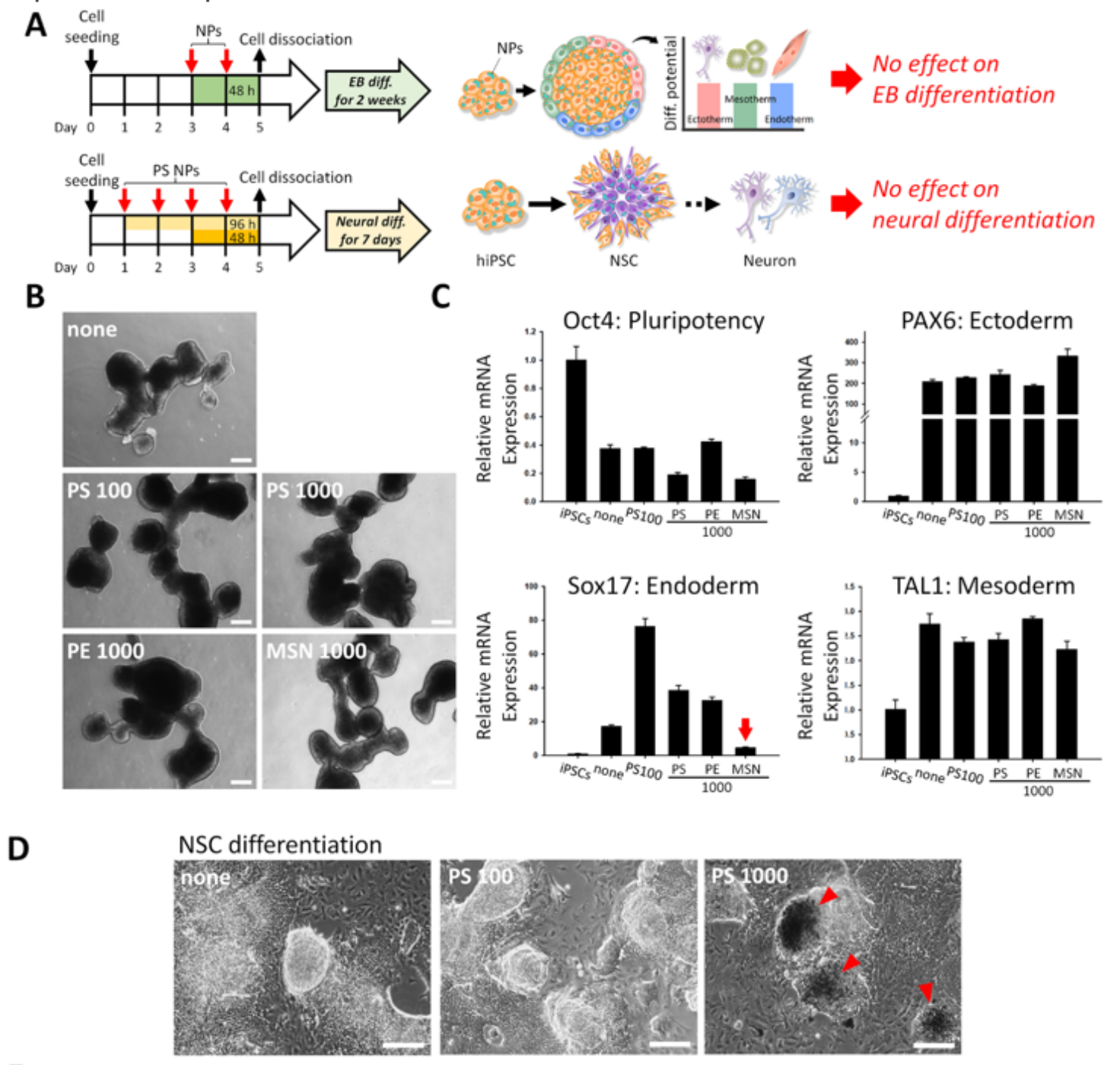

E
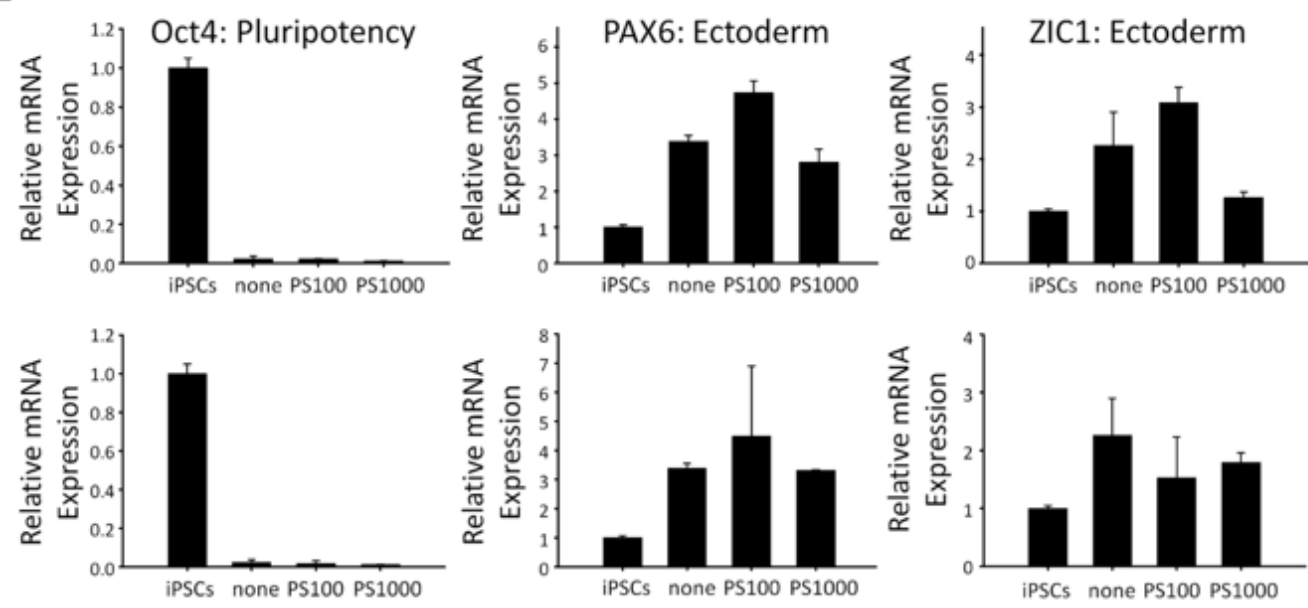

$48 \mathrm{~h}$

$96 \mathrm{~h}$

Figure 3 
Evaluation of EB formation, EB-mediated differentiation and direct neural differentiation ability of hiPSCs. EB, embryonic body, NPs, nanoplastics, hiPSCs, human induced pluripotent stem cells. (A) Schematic illustration of the experiment and long-term effects of NPs on EB and neural differentiation. Before EB formation, hiPSCs were pre-exposed to $100 \mu \mathrm{g} / \mathrm{mL}$ of NPs (PS 100, PS 1000, PE 1000, and MSN 1000, units are $\mathrm{nm}$.) for $48 \mathrm{~h}$. For neural differentiation, hiPSCs were pre-exposed to $100 \mu \mathrm{g} / \mathrm{mL}$ of PS 100 and PS 1000 for 48 and 96 h, respectively. (B) Morphology of 14-day-old EBs observed using optical microscopy. The inset depicts NP-treated EBs. The scale bar represents $200 \mu \mathrm{m}$. (C) Characterization of the effects of NPs (PS NPS, PE, and MSN) on the ability of EBs to spontaneously differentiate into the three germ lineages. Verification of the expression of the pluripotency marker (Oct4), ectoderm marker (PAX6), endoderm marker (Sox17), and mesoderm marker (TAL1) based on mRNA expression. Histogram values represent mean $\pm S D$. $(n=3)$ The condition where toxicity was observed has been indicated by a red arrow. (D) Morphology of 7-day-old neural stem cells (NSCs), generated through the differentiation of individual hiPSCs (exposed to PS NPs for $48 \mathrm{~h}$ ). The red arrows in the image of PS 1000 indicate the agglomeration of PS NPs. The scale bar represents $200 \mu \mathrm{m}$. (E) Characterization of NSCs based on the mRNA expression of the pluripotency marker (Oct4) and ectoderm markers (PAX6 and ZIC1). Histogram values represent mean $\pm S D$. $(n=3)$

1. Colony formation and self-renewal

No effect on colony formation

$>$ Possible inhibition of self-renewal capacity

- Growth inhibition

- Slight decrease in Nanog expression

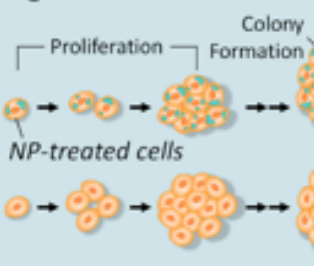

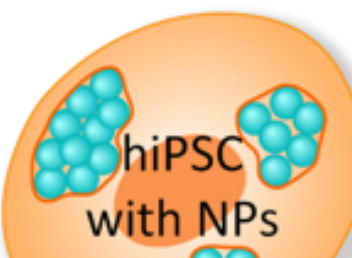

Pि
2. Pluripotency

$>$ No effect on EB formation

No effect on differentiation potential

- Spontaneous differentiation into three germ layers

- Direct differentiation into neuro-ectoderm lineage
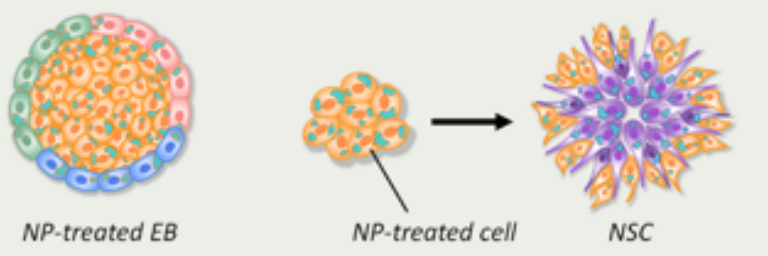

\section{Potential threat of NP accumulation in human}

\section{embryonic development}

\section{Figure 4}


Summary of the effects of NPs on hiPSCs. NPs, nanoplastics, hiPSCs, human induced pluripotent stem cells

\section{Supplementary Files}

This is a list of supplementary files associated with this preprint. Click to download.

- 3.SupportingInformationPFT.docx 